\title{
Modeling and Predicting Tumor Response in Radioligand Therapy
}

\author{
Peter Kletting ${ }^{1,2}$, Anne Thieme ${ }^{3}$, Nina Eberhardt ${ }^{1}$, Andreas Rinscheid ${ }^{1,2}$, Calogero D' Alessandria ${ }^{3}$, Jakob Allmann $^{3}$, \\ Hans-Jürgen Wester ${ }^{4}$, Robert Tauber ${ }^{5}$, Ambros J. Beer ${ }^{1}$, Gerhard Glatting ${ }^{1,2}$, and Matthias Eiber ${ }^{3}$ \\ ${ }^{I}$ Department of Nuclear Medicine, Ulm University, Ulm, Germany; ${ }^{2}$ Medical Radiation Physics, Department of Nuclear Medicine, \\ Ulm University, Ulm, Germany; ${ }^{3}$ Department of Nuclear Medicine, Klinikum Rechts der Isar, Technische Universität München, \\ Munich, Germany; ${ }^{4}$ Pharmaceutical Radiochemistry, Technische Universität München, Garching, Germany; and ${ }^{5}$ Department of \\ Urology, Klinikum Rechts der Isar, Technische Universität München, Munich, Germany
}

\begin{abstract}
The aim of this work was to develop a theranostic method that allows prediction of prostate-specific membrane antigen (PSMA)positive tumor volume after radioligand therapy (RLT) based on a pretherapeutic PET/CT measurement and physiologically based pharmacokinetic/pharmacodynamic (PBPK/PD) modeling at the example of RLT using ${ }^{177} \mathrm{Lu}$-labeled PSMA for imaging and therapy (PSMA I\&T). Methods: A recently developed PBPK model for ${ }^{177} \mathrm{Lu}-$ PSMA I\&T RLT was extended to account for tumor (exponential) growth and reduction due to irradiation (linear quadratic model). Data from 13 patients with metastatic castration-resistant prostate cancer were retrospectively analyzed. Pharmacokinetic/pharmacodynamic parameters were simultaneously fitted in a Bayesian framework to PET/CT activity concentrations, planar scintigraphy data, and tumor volumes before and after (6 wk) therapy. The method was validated using the leave-one-out Jackknife method. The tumor volume after therapy was predicted on the basis of pretherapy PET/CT imaging and PBPK/PD modeling. Results: The relative deviation of the predicted and measured tumor volume for PSMA-positive tumor cells (6 wk after therapy) was $1 \% \pm 40 \%$, excluding 1 patient (prostate-specific antigen-negative) from the population. The radiosensitivity for the prostate-specific antigenpositive patients was determined to be $0.0172 \pm 0.0084 \mathrm{~Gy}^{-1}$. Conclusion: To our knowledge, the proposed method is the first attempt to solely use PET/CT and modeling methods to predict the PSMA-positive tumor volume after RLT. Internal validation shows that this is feasible with an acceptable accuracy. Improvement of the method and external validation of the model is ongoing.
\end{abstract}

Key Words: PBPK/PD model; radioligand therapy; ${ }^{177}$ Lu-PSMA; tumor response

J Nucl Med 2019; 60:65-70

DOI: 10.2967/jnumed.118.210377

$\mathbf{R}$ ecently several studies have been published reporting the safety and efficacy of radioligand therapy (RLT) using ${ }^{177} \mathrm{Lu}-\mathrm{la}-$ beled prostate-specific membrane antigen (PSM)-specific peptides for the treatment of patients with metastatic castration-resistant prostate cancer (mCRPC) (1-4). Hematologic, renal parameters

Received Feb. 25, 2018; revision accepted May 8, 2018.

For correspondence or reprints contact: Peter Kletting, Department of Nuclear Medicine, Ulm University, D-89081 Ulm, Germany.

E-mail: peter.kletting@uniklinik-ulm.de

Published online May 10, 2018.

COPYRIGHT (C 2019 by the Society of Nuclear Medicine and Molecular Imaging. and changes in prostate-specific antigen (PSA) levels were investigated in most studies $(1-4)$. No study so far has investigated the relationship of the biologically effective dose (BED) and tumor volume changes. A dose-effect relationship is, however, a prerequisite for an improved therapy and adequate treatment planning. To establish such a relationship, a mathematic model, which describes both the pharmacokinetics and the pharmacodynamics (PD) (i.e., tumor growth and radiation effect) is needed. With such a model, tumor volume changes could be predicted on the basis of the injected activity (peptide amount and ligand properties). A first step toward that end was the development of a physiologically based pharmacokinetic (PBPK) model for ${ }^{177}$ Lu-labeled PSMA for imaging and therapy (PSMA I\&T) (5-7). The next logical step is to link the BED to tumor reduction.

The aim of this work was to develop a theranostic method that allows predicting tumor volume after RLT based on a pretherapeutic PET/CT and a PBPK/PD model. The model was developed using data (including PET/CT and planar images) of 13 patients with mCRPC treated with ${ }^{177}$ Lu-PSMA I\&T. A recently published PBPK model (7) was extended with a tumor growth and linear quadratic model. Pathophysiologic and radiobiologic parameters for 26 tumor lesions were estimated in a Bayesian framework (8). The Jackknife method $(9,10)$ was used for internal validation. The prediction accuracy of the model was determined by comparing the predicted and measured tumor volume $6 \mathrm{wk}$ after therapy. The prediction was based on the pretherapy PET/CT image and PBPK/ PD modeling.

\section{MATERIALS AND METHODS}

\section{Patient Data}

The patient data have been described elsewhere (4). In brief, the data of 13 patients (5) with mCRPC were included (4). All patients underwent the first-cycle ${ }^{177} \mathrm{Lu}$-PSMA I\&T RLT. The patients received $91.0 \pm 5.0$ nmol PSMA I\&T labeled with $7.3 \pm 0.3 \mathrm{GBq}$ of ${ }^{177} \mathrm{Lu}$. The mean age and body surface area were $70 \pm 6$ y and $2.0 \pm$ $0.12 \mathrm{~m}^{2}$, respectively. The institutional review board of the Technische Universität München approved the compassionate use of ${ }^{177} \mathrm{Lu}$-PSMA I\&T in mCRPC patients without other therapy options. All procedures performed in studies involving human participants were in accordance with the ethical standards of the institutional or national research committee and with the 1964 Helsinki Declaration and its later amendments or comparable ethical standards. For this retrospective study, informed consent was obtained from all individual participants and the retrospective analysis of the data was approved by the Ethics Committee of the Technical University Munich. 


\section{Planar Therapeutic Imaging and Image Processing}

Planar whole-body scintigraphy was performed as described previously (4). Two tumor lesions per patient with high uptake showing no considerable overlap with other tissue, that is, other lesions or high physiologic uptake, were investigated. In total, 20 bone and 6 softtissue metastases were analyzed (Table 1). The activity in the tumor region of interest included contributions from actual tumor and activity of the underlying muscle or adipose tissue. PBPK modeling was used to correct for underlying tissue activity ((5) and supplemental materials, section B, Equation B.1 [supplemental materials are available at http://jnm.snmjournals.org]). Three patients underwent posttherapy scintigraphy at 5 time points, 1 patient at 4 time points, and 9 patients at 3 time points (5). For this study, time-activity data of the kidneys, tumor, and total body were used (supplemental materials, section B, Supplemental Table B.1).

\section{PET/CT Imaging and Image Processing}

${ }^{68} \mathrm{Ga}$-PSMA-HBED-CC PET/CT for pre- and posttherapeutic staging was performed as described previously $(4,11)$. The average injected amount of PSMA HBED-CC and ${ }^{68} \mathrm{Ga}$ activity was $1.6 \pm 0.3$ nmol and $115 \pm 16 \mathrm{MBq}$, respectively. The PET/CT image before therapy was used to determine the tumor volume and the fraction of injected activity required for fitting. For tumor volume determination, SyngoVia (Syngo MMWP; Siemens Healthcare) was used. To estimate the volume of a single lesion and to determine the fraction of the tumor volume with PSMA-positive cells, a volume of interest with a $20 \%-50 \%$ of SUV $_{\max }$ isocontour adjusting the volume-of-interest optimal to the anatomic configuration of the lesion was drawn (4). The postprocessing software application TrueD (Siemens Healthcare) was used to derive the activity concentration of the kidney and 2 tumor lesions (tumor 1, tumor 2). Background activity correction was

TABLE 1

Patients Data and Measurements

\begin{tabular}{|c|c|c|c|c|c|c|c|}
\hline \multirow[b]{2}{*}{ Patient } & \multicolumn{2}{|c|}{ PSA (ng/mL) } & \multicolumn{3}{|c|}{ Time difference (d) } & \multicolumn{2}{|c|}{$\begin{array}{l}\text { PET/CT tumor } \\
\text { volume (mL) }\end{array}$} \\
\hline & Therapy & $\begin{array}{l}\text { Second } \\
\text { PET/CT }\end{array}$ & $\begin{array}{l}\text { First PET/CT } \\
\text { to therapy }\end{array}$ & $\begin{array}{c}\text { Therapy to second } \\
\text { PET/CT }\end{array}$ & Location & $\begin{array}{l}\text { Before } \\
\text { therapy }\end{array}$ & $\begin{array}{l}\text { After } \\
\text { therapy }\end{array}$ \\
\hline \multirow[t]{2}{*}{$\mathrm{P} 1$} & 513 & 259 & 20 & 42 & Bone & 9.4 & 7.7 \\
\hline & & & & & Bone & 19 & 12 \\
\hline \multirow[t]{2}{*}{ P2 } & 49 & 29 & 21 & 42 & $\mathrm{LN}^{*}$ & 2.1 & 1.2 \\
\hline & & & & & Liver & 4.2 & 3.9 \\
\hline \multirow[t]{2}{*}{ P3 } & 31 & 56 & 13 & 42 & Bone & 14 & 19 \\
\hline & & & & & Bone & 52 & 102 \\
\hline \multirow[t]{2}{*}{ P4 } & 97 & 69 & 21 & 42 & Bone & 14 & 15 \\
\hline & & & & & Bone & 31 & 29 \\
\hline \multirow[t]{2}{*}{ P5 } & 47 & 28 & 18 & 41 & Bone & 26 & 12 \\
\hline & & & & & Bone & 17 & 16 \\
\hline \multirow[t]{2}{*}{ P6 } & 2,905 & 2,310 & 8 & 42 & Bone & 81 & 79 \\
\hline & & & & & Bone & 92 & 96 \\
\hline \multirow[t]{2}{*}{ P7 } & 0.23 & 0.07 & 35 & 41 & Bone & 48 & 32 \\
\hline & & & & & Lung & 37 & 4.6 \\
\hline \multirow[t]{2}{*}{ P8 } & 1,193 & 181 & 12 & 41 & Bone & 5.7 & 3.3 \\
\hline & & & & & Bone & 19 & 14 \\
\hline \multirow[t]{2}{*}{ P9 } & 2,547 & 2,158 & 63 & 41 & Bone & 15 & 32 \\
\hline & & & & & Bone & 8.7 & 17 \\
\hline \multirow[t]{2}{*}{$\mathrm{P} 10$} & 2,471 & 4,194 & 15 & 41 & Bone & 1.9 & 2.4 \\
\hline & & & & & Bone & 5.3 & 6.4 \\
\hline \multirow[t]{2}{*}{ P11 } & 7 & 3.9 & 20 & 41 & Bone & 0.33 & 0.33 \\
\hline & & & & & $\mathrm{LN}^{*}$ & 1.2 & 1.7 \\
\hline \multirow[t]{2}{*}{$\mathrm{P} 12$} & 161 & 139 & 21 & 38 & Bone & 9.9 & 11 \\
\hline & & & & & Bone & 8.7 & 11 \\
\hline \multirow[t]{2}{*}{$\mathrm{P} 13$} & 133 & 113 & 19 & 38 & $\mathrm{LN}^{*}$ & 5.9 & 10 \\
\hline & & & & & $\mathrm{LN}^{*}$ & 2.0 & 2.1 \\
\hline Mean & 781 & 939 & 22 & 41 & & 18 & 24 \\
\hline SD & 1,112 & 1,374 & 14 & 1 & & 22 & 30 \\
\hline Median & 133 & 113 & 20 & 41 & & 12 & 12 \\
\hline Range & $0.23-2,905$ & $0.07-4,194$ & $8-63$ & 38-42 & & $0.33-92$ & $0.33-102$ \\
\hline
\end{tabular}


conducted within the PBPK model (supplemental materials, section B, Equations B.2-3).

The activity and volume of all other tumor lesions-named REST tumor in the following - were obtained by adding up all lesions slice by slice as described in the supplemental materials, section B, Equation B.4.

\section{PBPK Model Structure}

SAAMII and Popkinetics (12) (version 2.2; The $\varepsilon$-Group) were used for modeling and fitting. For the pharmacokinetic part of the model, a recently developed whole-body PBPK model was applied $(5,6)$. The PBPK model includes all relevant biologic mechanisms such as blood flow, diffusion, PSMA-specific binding, and internalization as well as excretion.

\section{Tumor Effect Model}

Tumor growth and reduction was modeled using exponential growth and the linear quadratic model for the surviving fraction. Considering the slow net growth rates for prostate cancer and the time interval between the pretherapeutic PET/CT and therapy ( $\leq 3 \mathrm{wk}$ in $11 / 13$ patients) and posttherapy PET/CT (6 wk), simple exponential growth is a good approximation to Gompertzian growth or other growth models. The volume of the tumor $V_{\mathrm{TU}, \text { total, } 0}$ was thus described:

$$
\begin{array}{r}
V_{\mathrm{TU}, \text { total }}(t)=V_{\mathrm{TU}, \text { total }, 0} \times \mathrm{e}^{\left(\lambda_{\mathrm{g}} \times t-\alpha_{\mathrm{TU}} \times B E D_{\mathrm{TU}}\right)} \\
B E D_{\mathrm{TU}}=D_{\mathrm{TU}} \times\left(1+\frac{G_{\mathrm{TU}}}{\alpha_{\mathrm{TU}} / \beta_{\mathrm{TU}}} \times D_{\mathrm{TU}}\right)
\end{array}
$$

where $t$ is the elapsed time starting from the pretherapeutic PET/CT, $V_{\mathrm{TU}, \text { total }, 0}$ is the volume of the first PET/CT, $\lambda_{g}$ is the net growth rate (bone lesions: $5.1 \times 10^{-6} \mathrm{~min}^{-1}$; soft-tissue lesions: $3.8 \times 10^{-6} \mathrm{~min}^{-1}$ ) as

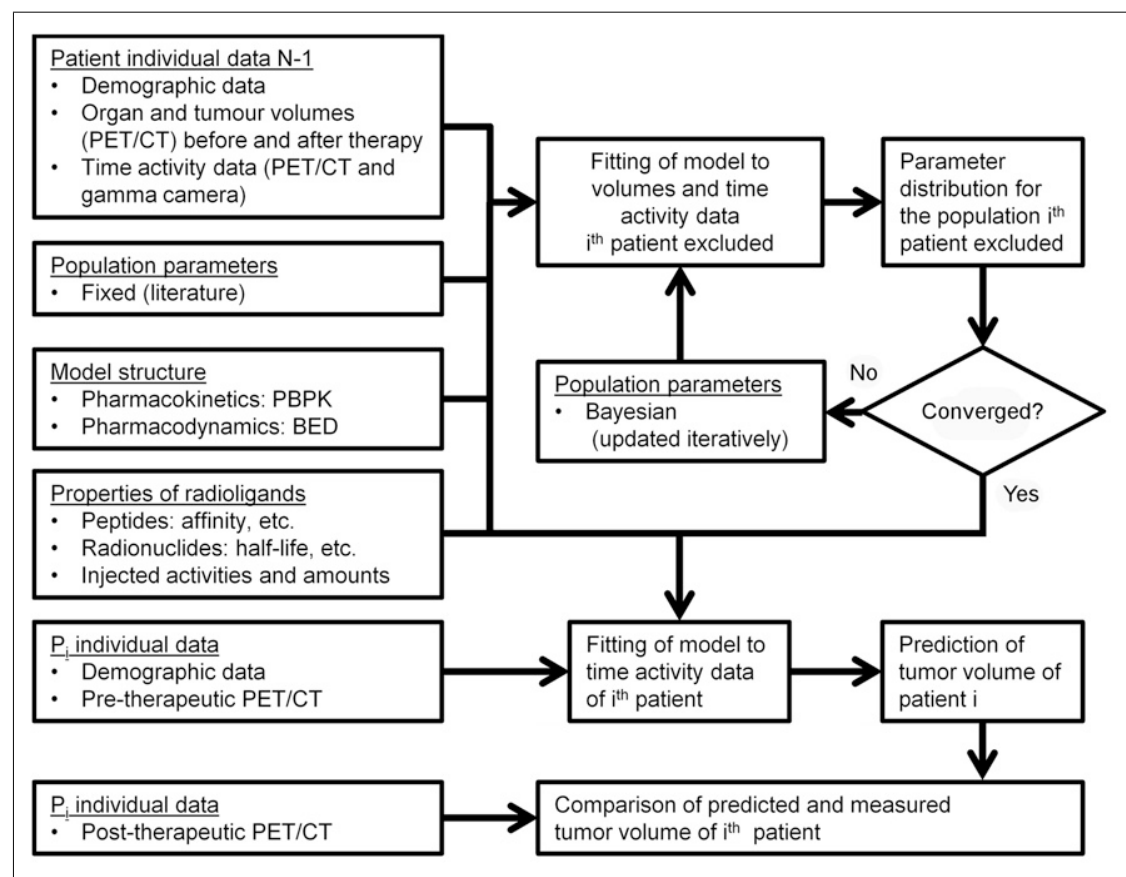

FIGURE 1. Model fitting and prediction of tumor volume after treatment. Iterative fitting approach was used to determine Bayesian parameters for investigated population. On the basis of the PBPK model, population parameters (as fixed or Bayesian information) and pretherapeutic PET of excluded patient, volume after treatment was predicted and compared with measured values. This was conducted for all $(n=13)$ patients. reported by Berges et al. (13) for androgen-independent cells, $B E D_{\mathrm{TU}}$ is the biologically effective dose, and $\alpha_{\mathrm{TU}}$ is the intrinsic radiosensitivity of PSMA-positive tumor cells. The radiosensitivity $\alpha_{\mathrm{TU}}$ is fitted. It represents rather an effective value as it is not known to which extent radiation (lethally) damaged cells still express PSMA until they die.

The volumes determined by the posttherapy PET/CT were included as measurement data (assuming a relative error of 10\%) for the fitting process. The elapsed times from the first PET/CT to therapy and to the second PET/CT were 8-63 d and 38-42 d, respectively (Table 1 ). The $\alpha_{\mathrm{TU}} / \beta_{\mathrm{TU}}$ ratio $(1.49 \mathrm{~Gy})$ and repair rate $\mu_{\mathrm{TU}}\left(0.0061 \mathrm{~min}^{-1}\right)$ were assumed to equal those determined for brachytherapy for primary prostate cancer (14) and were incorporated as fixed values. The Lea-Catcheside factor $G$ of each tumor lesion was numerically determined within the PBPK model according to (15):

$$
\begin{aligned}
G_{\mathrm{TU}}(T)= & \frac{2}{D_{\mathrm{TU}}^{2}} \times \int_{0}^{T} \dot{D}_{\mathrm{TU}}(t) \mathrm{d} t \times \int_{0}^{t} \dot{D}_{\mathrm{TU}}(\omega) \\
& \times e^{-\mu_{\mathrm{TU}}(t-\omega)} \mathrm{d} \omega .
\end{aligned}
$$

For the calculation of the absorbed dose, only the self-dose was considered:

$$
\dot{D}_{\mathrm{TU}}(t)=A_{\mathrm{TU}}(t) \times S_{\mathrm{TU} \leftarrow \mathrm{TU}}=A_{\mathrm{inj}} \times a_{\mathrm{TU}}(t) \times S_{\mathrm{TU} \leftarrow \mathrm{TU}} \quad \text { Eq. } 4
$$

$$
D_{\mathrm{TU}}(T)=\int_{0}^{T} \dot{D}_{\mathrm{TU}}(t) \mathrm{d} t=A_{\mathrm{inj}} \times \tilde{a}_{\mathrm{TU}}(T) \times S_{\mathrm{TU} \leftarrow \mathrm{TU}}
$$

The dose factors for the tumor lesions were derived from OLINDA/ EXM (16) data as described in the supplemental materials, section A, Table B. The dose factors were assumed to be constant during therapy.

\section{Population Fitting}

For parameter estimation, an iterative fitting approach (Fig. 1) (9) was used. To determine the population parameters, the model parameters for each patient were simultaneously fitted to the activity concentration of the pretherapeutic PET, the time-activity data during therapy, and the tumor volumes. Thus, 10 adjustable parameters (with Bayesian term) were fitted to a minimum of 17 data points. Tumor growth (exponential) and reduction (linear quadratic model), different peptide properties (e.g., molecular size), and injected amounts for PET/CT and therapy were considered.

For internal validation the leave-one-out Jackknife method was used. For that purpose, 13 population parameter distributions were determined using the method described above leaving out 1 patient each time. The quality of the fits was checked according to Kletting et al. (17).

\section{Individual Fitting and Prediction Accuracy}

To internally validate the prediction power of the model, for each patient, the predicted and the actual tumor volume were compared. For that, the blood flow and receptor density of 
each individual patient ( 7 parameters) were fitted with Bayesian information (of the 12 other patients) solely to the pretherapeutic PET/CT data ( 1 data point per investigated region of interest). The release rates and the radiosensitivity $\alpha_{\mathrm{TU}}$ were fixed to the mean value of the leave-one-out population parameter. Fitting in a Bayesian framework allows having more adjustable parameters than number of samples.

Thus, the predictions of the posttherapy tumor volume was based on the PBPK/PD model, the population parameters of the 12 other patients (as Bayesian information), and the pretherapeutic PET/CT of each patient. The prediction accuracy of the tumor volume was defined as the relative deviation, $R D$, of the simulated and measured tumor volume, $V_{\mathrm{TU}, \text { total,PETCT2}}$, after therapy at the time of the second $\mathrm{PET} / \mathrm{CT}, t_{\mathrm{PETCT} 2}$.

$$
\begin{aligned}
R D_{\mathrm{TU}, \text { volume }}= & \frac{V_{\mathrm{TU}, \text { total }, 0} \times \mathrm{e}^{\left(\lambda_{\mathrm{g}} \times t_{\mathrm{PETCT} 2}-\alpha_{\mathrm{TU}} \times B E D_{\mathrm{TU}}\right)}-V_{\mathrm{TU}, \text { total }, \text { PETCT2 }}}{V_{\mathrm{TU}, \text { total }, \text { PETCT2 }}} \\
& \times 100 \% .
\end{aligned}
$$

\section{Predicted BEDs and Tumor Volume Changes}

The BED was predicted using the PBPK/PD model, the jackknife population parameters (including the $\alpha_{\mathrm{TU}}$ ), and the pretherapy PET/ CT data. To demonstrate the relationship of tumor volume reduction and BED, the predicted relative reduction of the tumor volume was calculated for each lesion. The tumor volume at the beginning of therapy was used as reference point, to normalize for varying time intervals between the pretherapeutic PET and therapy.

\section{RESULTS}

\section{Population Fitting}

The radiosensitivities $\alpha_{\mathrm{TU}}$ provided in Table 2 show the averaged values and pertaining SD (leaving out the corresponding patient) after convergence of the iterative fitting process. The Jackknife method showed that patient 7 (PSA-negative) considerably changed the population values (Table 2). Thus, this patient was removed and in a second step the Jackknife method was again applied to the reduced population. The average radiosensitivity $\alpha_{\mathrm{TU}}$ of the 13 investigated patients and the population without patient 7 was estimated to be $0.022 \pm 0.022 \mathrm{~Gy}^{-1}$ and $0.0172 \pm$ $0.0084 \mathrm{~Gy}^{-1}$, respectively. For the reduced patient population, visual inspection showed excellent fits, except for patient 5, tumor 2. The coefficient of determination $R^{2}$ (supplemental materials, section C, Table 2) was greater than 0.8 for all curves in all patients, except for patient 5, tumor $1\left(R^{2}=0.77\right)$ and tumor 2 $\left(R^{2}=0.40\right)$, and for patient 3 , total body $\left(R^{2}=0.73\right)$. A typical fit and the one with the lowest $R^{2}$ are depicted in supplemental materials, section C, Figure C.1. All fits yielded coefficients of variation (relative standard errors) less than $50 \%$ for any estimated parameter, except $\alpha_{\mathrm{TU}}$ of patients $3(52 \%)$ and $9(56 \%)$. Elements of the correlation matrix were between -0.76 and 0.71 . The values of the estimated parameters are in a physiologically reasonable range, that is, comparing favorably to literature values (supplemental materials, section $\mathrm{C}$, Table 1).

\section{Individual Fitting and Prediction Accuracy}

Visual inspection showed excellent fits. The coefficients of variation were less than $27 \%$ for all blood flows and less than $71 \%$ for the tumor receptor densities. Elements of the correlation matrix were between -0.88 and 0.71 .

The mean relative deviation of the measured and predicted tumor volume in the investigated population (without patient 7) was determined to be $1 \% \pm 40 \%$ (Table 2; Fig. 2).

TABLE 2

\begin{tabular}{|c|c|c|c|c|c|c|c|c|c|c|c|c|c|}
\hline \multirow[b]{3}{*}{ Patient } & \multirow{2}{*}{\multicolumn{2}{|c|}{$\mathrm{RD}(\%)$}} & \multirow[b]{3}{*}{$\mathrm{a}_{\mathrm{TU}}\left(\mathrm{Gy}^{-1}\right), \mathrm{T} 1, \mathrm{~T} 2$} & \multirow{2}{*}{\multicolumn{2}{|c|}{$\begin{array}{c}\text { D } \\
\text { predicted }^{*} \text { (Gy) }\end{array}$}} & \multirow{2}{*}{\multicolumn{2}{|c|}{$\begin{array}{c}\text { BED } \\
\text { predicted }^{\star}\left(\mathrm{Gy}_{\alpha / \beta}\right)\end{array}$}} & \multicolumn{4}{|c|}{$\mathrm{V}_{\text {TU,Tumor }}(\mathrm{mL})$} & \multirow{2}{*}{\multicolumn{2}{|c|}{$\begin{array}{c}\text { Reduction } \\
\text { predicted }^{\star}(\%)\end{array}$}} \\
\hline & & & & & & & & \multicolumn{2}{|c|}{$\begin{array}{c}\text { Therapy } \\
\text { predicted }^{*}\end{array}$} & \multicolumn{2}{|c|}{$\begin{array}{l}\text { Posttherapy } \\
\text { predicted }^{*}\end{array}$} & & \\
\hline & $\mathrm{T} 1$ & $\mathrm{~T} 2$ & & $\mathrm{~T} 1$ & $\mathrm{~T} 2$ & $\mathrm{~T} 1$ & T2 & $\mathrm{T} 1$ & T2 & $\mathrm{T} 1$ & $\mathrm{~T} 2$ & $\mathrm{~T} 1$ & $\mathrm{~T} 2$ \\
\hline $\mathrm{P} 1$ & -17 & 57 & $0.0176 \pm 0.0091$ & 28 & 17 & 48 & 24 & 11 & 22 & 6.4 & 19 & 41 & 11 \\
\hline $\mathrm{P} 2$ & 34 & -17 & $0.0174 \pm 0.0091$ & 24 & 24 & 36 & 36 & 2.3 & 4.7 & 1.6 & 3.2 & 31 & 31 \\
\hline P3 & -18 & -43 & $0.0161 \pm 0.0080$ & 13 & 13 & 17 & 16 & 15 & 57 & 15 & 59 & -0.95 & -2.4 \\
\hline $\mathrm{P} 4$ & -4.7 & 10 & $0.0152 \pm 0.0066$ & 15 & 16 & 21 & 23 & 16 & 36 & 15 & 32 & 7.9 & 11 \\
\hline P5 & 120 & 13 & $0.0186 \pm 0.0080$ & 19 & 17 & 27 & 24 & 30 & 20 & 27 & 18 & 10 & 6.3 \\
\hline P6 & 30 & 23 & $0.0172 \pm 0.0090$ & 6.6 & 6.2 & 7.7 & 7.1 & 85 & 97 & 103 & 118 & -21 & -21 \\
\hline $\mathrm{P} 7$ & - & - & - & - & - & - & - & - & - & - & - & - & - \\
\hline P8 & 49 & 28 & $0.0165 \pm 0.0086$ & 21 & 20 & 31 & 30 & 6.3 & 21 & 4.9 & 17 & 22 & 19 \\
\hline P9 & -26 & -15 & $0.0187 \pm 0.0076$ & 13 & 11 & 17 & 14 & 24 & 14 & 24 & 14 & 1.8 & -3.2 \\
\hline P10 & -23 & -15 & $0.0182 \pm 0.0086$ & 17 & 16 & 25 & 21 & 2.2 & 6.0 & 1.9 & 5.5 & 14 & 8.3 \\
\hline P11 & 3.0 & -32 & $0.0182 \pm 0.0086$ & 17 & 17 & 25 & 23 & 0.38 & 1.4 & 0.34 & 1.1 & 11 & 16 \\
\hline P12 & -3.9 & -12 & $0.0174 \pm 0.0090$ & 15 & 15 & 21 & 21 & 12 & 10 & 11 & 10 & 5.6 & 6.1 \\
\hline $\mathrm{P} 13$ & -58 & -66 & $0.0163 \pm 0.0083$ & 24 & 39 & 36 & 74 & 6.8 & 2.2 & 4.3 & 0.72 & 36 & 68 \\
\hline Mean & 1 & & 0.0172 & 17.7 & & 26 & & 21 & & 21 & & 13 & \\
\hline SD & 40 & & 0.0084 & 6.9 & & 14 & & 25 & & 30 & & 19 & \\
\hline
\end{tabular}

Predicted Therapeutic and Posttherapeutic Quantities Based on Pretherapy PET/CT

${ }^{*}$ Predicted $=$ these values were determined (for the beginning of therapy and 6 wk after therapy) using the pretherapeutic PET/CT/and the PBPK/PD model.

$\mathrm{RD}=$ relative deviation of predicted and measured tumor volumes 6 wk after therapy; $\mathrm{a}_{\mathrm{TU}}=$ radiosensitivities estimated using populations fits and Jackknife method for PSA-positive patients; $\mathrm{D}=$ absorbed dose; $\mathrm{V}_{\mathrm{TU}}$,Tumor $=$ tumor volume; Reduction = predicted reduction for $6 \mathrm{wk}$ after therapy relative to the beginning of therapy; T1 and T2 = tumor 1 and tumor 2 . 


\section{Predicted BEDs and Tumor Volume Changes}

Table 2 shows the BEDs, the tumor volumes at the beginning of therapy, and the predicted tumor volumes $6 \mathrm{wk}$ after therapy. The relationship between the predicted BED and the predicted tumor volume reduction after therapy is depicted in Figure 3.

\section{DISCUSSION}

In this work, we developed a method to predict PSMA-positive tumor volume after ${ }^{177} \mathrm{Lu}$-PSMA I\&T RLT using the pretherapeutic PET/CT and a PBPK/PD model. Data of 13 patients with mCRPC were used. Pharmacokinetic parameters and the radiosensitivity $\alpha_{\mathrm{TU}}$ were estimated using a PBPK/PD, the time-activity data (PET/CT and planar images), and tumor volumes of 13 patients (26 tumor lesions). Data from literature values for growth of androgen-independent prostatic cancer cells (13) and radiobiologic parameters $\left(\alpha_{\mathrm{TU}} / \beta_{\mathrm{TU}}\right.$ and $\left.\mu_{\mathrm{TU}}\right)$ known from brachytherapy (14) were used. The values obtained (PSA-positive patients) for radiosensitivity $\alpha_{\mathrm{TU}}\left(0.0172 \pm 0.0084 \mathrm{~Gy}^{-1}\right)$ are 2.3 -fold lower than the mean values determined for primary tumor brachytherapy (14). For internal validation, the leave-one-out Jackknife method was used. The difference of the measured and predicted PSMApositive tumor volume (accessible to the peptide) was $1 \% \pm 40 \%$, excluding the PSA-negative patient. In this patient, the radiosensitivity $\alpha_{\mathrm{TU}}$ was considerably larger $\left(0.068 \mathrm{~Gy}^{-1}\right)$.

Here, planar imaging was used to identify population parameters, which were then included as Bayesian or fixed parameters for individual fitting, prediction, and validation. Population parameters were obtained by simultaneous fitting of 3-dimensional (pretherapeutic PET/CT) and 2-dimensional (planar) data in combination with sophisticated corrections methods. The more accurate PET/CT allowed correcting data for the first hours of uptake, whereas the information for later time points solely stems from planar imaging. Therefore, we believe that the release rates may represent the most uncertain parameters. Undoubtedly, it would be desirable to have population values with lower SD. This could be achieved with measurements that are more accurate and with the creation of subpopulations depending, for example, on the PSA level.

Therefore, the focus of future work regarding this presented model approach should be on analyzing a larger patient group in

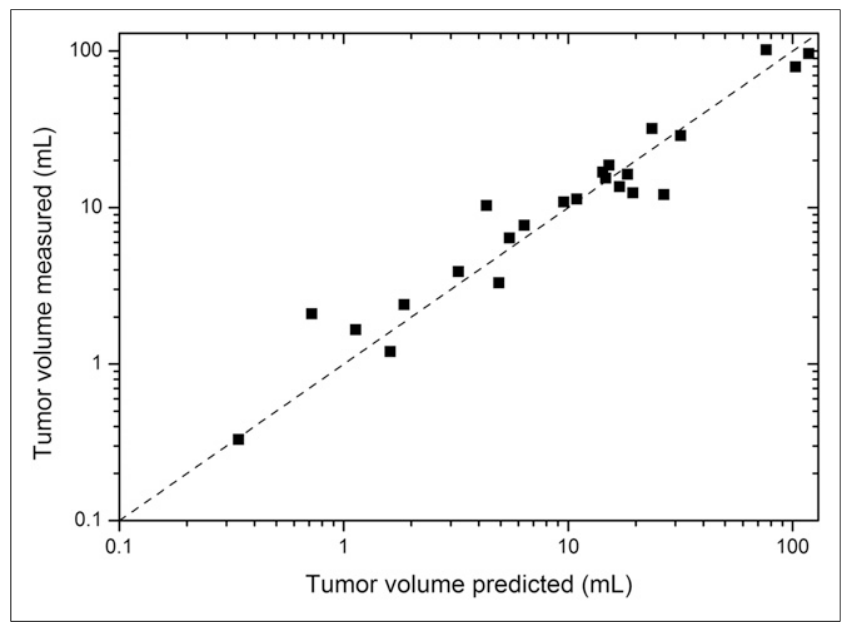

FIGURE 2. Predicted versus measured tumor volume. Prediction is based on PBPK/PD model, pretherapeutic measurement, and population parameters.

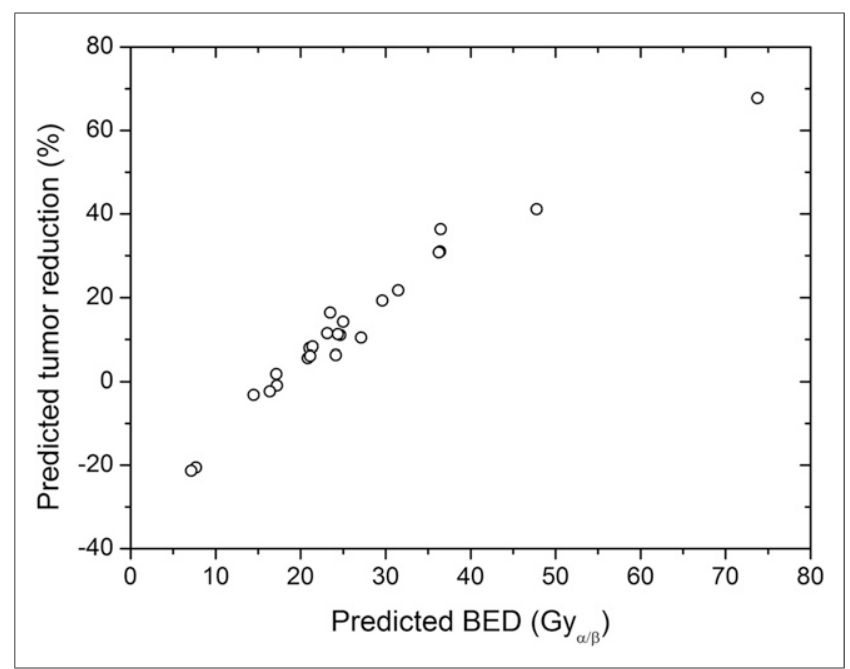

FIGURE 3. Predicted tumor volume reduction versus predicted BED. Predictions are based on PBPK/PD model, pretherapeutic measurement, and population parameters. Clear dose-effect (i.e., BED-volume change) relationship is visible. Note that for each patient different radiosensitivity was used. In addition, for bone and soft-tissue metastases different growth rates were assumed.

whom SPECT/CT imaging was conducted and including more a priori information of each individual. Planar imaging during therapy is still standard practice although SPECT/CT data might improve the accuracy of the estimated pharmacokinetic parameters. Although we have included 3-dimensional information using the pretherapeutic PET/CT for the fitting process, when 3-dimensional imaging during therapy is used more lesions per patient can be investigated, as the PBPK model allows correcting for overlaying normal tissue but not for other lesions. More soft-tissue lesions would be desirable, as it is inherently challenging to determine volume changes in bone metastases. However, when both were used, the PET and the CT information reduced the inaccuracy. An error of $10 \%$ to account for these uncertainties arising was assigned. Further error in the calculation of the absorbed dose and BED is introduced by assuming the tumor being a sphere, the same radiobiologic parameters for all lesions, and a constant growth rate over the whole time period. SPECT/CT data during therapy will help to reduce the number of assumptions. However, on the basis of our results it can be concluded that the assumptions made in this study were useful for the investigated population.

Furthermore, we conducted the population study including patients with a large variation in PSA levels. The prediction accuracy would probably further increase using subpopulations with similar PSA levels and treatment histories. More sophisticated tumor dynamic models including compartments for various conditions of tumor cells (vital, necrotic, lethally damaged) (18) might improve the overall understanding of the treatment effects and the relationships of the radiosensitivity and PSA or perhaps the overall tumor load.

As this work focuses on the tumor dose-effect relation, we did not define a clinical endpoint for dose effect on the kidneys and salivary glands. Future work will include treatment effect for normal tissue. Before applying this approach for a full in silico clinical trial, further validation and extension of our model is needed investigating the link between a PBPK/PD model and clinical outcome parameters (e.g., overall survival). 
External validation, using the model and the population parameters to predict the volume changes in a different patient group, is planned.

\section{CONCLUSION}

We present a method to model and predict tumor response in RLT. The volume of PSMA-positive tumor tissue (6 wk after therapy) was predicted on the basis of the pretherapeutic PET/CT and a PBPK/PD model. The relative deviation of the predicted and measured tumor volume was $1 \% \pm 40 \%$. Future work including a refined model, definition of subpopulations, and use of SPECT/CT data will help to improve further the prediction accuracy of the model.

\section{DISCLOSURE}

We gratefully acknowledge grants by the "Deutsche Forschungsgemeinschaft" (German Research Foundation, KL2742/2-1, BE4393/1-1, GL236/11-1, SFB824 Project B11). No other potential conflict of interest relevant to this article was reported.

\section{REFERENCES}

1. Kratochwil C, Giesel FL, Stefanova M, et al. PSMA-targeted radionuclide therapy of metastatic castration-resistant prostate cancer with ${ }^{177} \mathrm{Lu}$-labeled PSMA617. J Nucl Med. 2016;57:1170-1176.

2. Yadav MP, Ballal S, Tripathi M, et al. Lu-177-DKFZ-PSMA-617 therapy in metastatic castration resistant prostate cancer: safety, efficacy, and quality of life assessment. Eur J Nucl Med Mol Imaging. 2017;44:81-91.

3. Rahbar K, Ahmadzadehfar H, Kratochwil C, et al. German multicenter study investigating Lu-177-PSMA-617 radioligand therapy in advanced prostate cancer patients. J Nucl Med. 2017;58:85-90.

4. Okamoto S, Thieme A, Allmann J, et al. Radiation dosimetry for Lu-177-PSMA I\&T in metastatic castration-resistant prostate cancer: absorbed dose in normal organs and tumor lesions. J Nucl Med. 2017;58:445-450.
5. Nusrat JB, Thieme A, Eberhardt N, et al. The effect of total tumor volume on the biologically effective dose of tumor and kidneys for ${ }^{177} \mathrm{Lu}$-labelled PSMA peptides. J Nucl Med. 2018;59:929-933.

6. Kletting P, Kull T, Maass C, et al. Optimized peptide amount and activity for ${ }^{90} \mathrm{Y}-$ labeled DOTATATE therapy. J Nucl Med. 2016;57:503-508.

7. Kletting P, Schuchardt C, Kulkarni HR, et al. Investigating the effect of ligand amount and injected therapeutic activity: a simulation study for ${ }^{177} \mathrm{Lu}$-labeled PSMA-targeting peptides. PLoS One. 2016;11:e0162303.

8. Krauss M, Tappe K, Schuppert A, Kuepfer L, Goerlitz L. Bayesian population physiologically-based pharmacokinetic (PBPK) approach for a physiologically realistic characterization of interindividual variability in clinically relevant populations. PLoS One. 2015;10:e0139423.

9. Maaß C, Kletting P, Bunjes D, Mahren B, Beer AJ, Glatting G. Population-based modeling improves treatment planning before Y-90-labeled anti-CD66 antibody radioimmunotherapy. Cancer Biother Radiopharm. 2015;30:285-290.

10. Glatting G, Kletting P, Reske SN, Hohl K, Ring C. Choosing the optimal fit function: comparison of the Akaike information criterion and the F-test. Med Phys. 2007;34:4285-4292.

11. Eiber M, Maurer T, Souvatzoglou M, et al. Evaluation of hybrid Ga-68-PSMA ligand PET/CT in 248 Patients with biochemical recurrence after radical prostatectomy. J Nucl Med. 2015;56:668-674.

12. Barrett PH, Bell BM, Cobelli C, et al. SAAM II: simulation, analysis, and modeling software for tracer and pharmacokinetic studies. Metabolism. 1998;47: 484-492.

13. Berges RR, Vukanovic J, Epstein JI, et al. Implication of cell kinetic changes during the progression of human prostatic cancer. Clin Cancer Res. 1995;1: 473-480.

14. Fowler J, Chappell R, Ritter M. Is alpha/beta for prostate tumors really low? Int J Radiat Oncol Biol Phys. 2001;50:1021-1031.

15. Konijnenberg M. From imaging to dosimetry and biological effects. $Q \mathrm{~J} \mathrm{Nucl}$ Med Mol Imaging. 2011;55:44-56.

16. Stabin MG, Sparks RB, Crowe E. OLINDA/EXM: the second-generation personal computer software for internal dose assessment in nuclear medicine. $\mathrm{J} \mathrm{Nucl}$ Med. 2005;46:1023-1027.

17. Kletting P, Schimmel S, Kestler HA, et al. Molecular radiotherapy: the NUKFIT software for calculating the time-integrated activity coefficient. Med Phys. 2013; 40:102504.

18. Chvetsov AV, Dong L, Palta JR, Amdur RJ. Tumor volume simulation during radiotherapy for head and neck cancer using a four level cell population model. Int J Radiat Oncol Biol Phys. 2009;75:595-602. 